\title{
Simulating Scintillator Light Collection Using Measured Optical Reflectance
}

\author{
Martin Janecek, Member, IEEE, and William W. Moses, Fellow, IEEE
}

\begin{abstract}
To accurately predict the light collection from a scintillating crystal through Monte Carlo simulations, it is crucial to know the angular distribution from the surface reflectance. Current Monte Carlo codes allow the user to set the optical reflectance to a linear combination of backscatter spike, specular spike, specular lobe, and Lambertian reflections. However, not all light distributions can be expressed in this way. In addition, the user seldom has the detailed knowledge about the surfaces that is required for accurate modeling. We have previously measured the angular distributions within BGO crystals and now incorporate these data as look-up-tables (LUTs) into modified Geant4 and GATE Monte Carlo codes. The modified codes allow the user to specify the surface treatment (ground, etched, or polished), the attached reflector (Lumirror ${ }^{\circledR}$, Teflon ${ }^{\circledR}$, ESR film, Tyvek $^{\circledR}$, or TiO paint), and the bonding type (air-coupled or glued). Each LUT consists of measured angular distributions with $4^{\circ}$ by $5^{\circ}$ resolution in theta and phi, respectively, for incidence angles from $0^{\circ}$ to $90^{\circ}$ degrees, in $1^{\circ}$-steps. We compared the new codes to the original codes by running simulations with a $3 \times 10 \times 30 \mathrm{~mm}^{3}$ BGO crystal coupled to a PMT. The simulations were then compared to measurements. Light output was measured by counting the photons detected by the PMT with the $3 \times 10,3 \times 30$, or $10 \times 30 \mathrm{~mm}^{2}$ side coupled to the PMT, respectively. Our new code shows better agreement with the measured data than the current Geant 4 code. The new code can also simulate reflector materials that are not pure specular or Lambertian reflectors, as was previously required. Our code is also more user friendly, as no detailed knowledge about the surfaces or light distributions is required from the user.
\end{abstract}

Index Terms-Lambertian reflection, light collection, Monte Carlo methods

\section{INTRODUCTION}

$\mathrm{O}$ PTICAL Monte Carlo simulations are frequently used to predict the light distribution in scintillation crystals. For these simulations to be accurate, the surface characteristics have to be well understood. Current Monte Carlo simulation software, e.g., DETECT [1], [2], Litrani [3], Geant4 [4], [5], and GATE [6-9], are all based on mathematic models where the most advanced surface light reflection models can be

Manuscript received June 9, 2009; revised September 25, 2009; accepted January 28, 2010. Date of current version June 16, 2010. This work was supported by the National Nuclear Security Administration, Office of Defense Nuclear Nonproliferation, Office of Nuclear Nonproliferation Research and Engineering (NA-22) of the U.S. Department of Energy under Contract No. DE-AC02-05CH11231 and Grant NNSA LB06-316-PD05 / NN2001000.

The authors are with the Lawrence Berkeley National Laboratory, Berkeley, CA 94720 USA (email: mjanecek@lbl.gov; wwmoses@lbl.gov). described as a linear combination of backscatter spike, specular spike, specular lobe, and Lambertian reflections, and the attached reflector can be set as a either purely specular or purely Lambertian reflector. We have previously demonstrated that not all light distributions can be described in this way [10], [11]. In particular, some commonly used reflector materials, e.g., Tyvek ${ }^{\circledR}$, Lumirror $^{\circledR}$, and Melinex ${ }^{\circledR}$, have reflection distributions that are not linear combinations of these components. In addition, the user seldom has the detailed knowledge about the surface and the reflector to accurately set up a simulation with the required parameters for an accurate simulation. For example, GATE and Geant4 require the user to set the parameter sigmaalpha, which describes the angular distribution of the micro-facets that make up the macro-surface.

The aim of this work is to improve the accuracy of existing optical Monte Carlo simulations by using measured data from BGO reflectance measurements [11]. In addition, the usability of the current codes is improved, as the user does not need the detailed knowledge of surface roughness, standard deviation of reflected light spread, etc. to run the codes. We incorporated our measured data into the GATE and Geant4 simulation toolkits, since these codes are widely used for many applications, including medical imaging, high energy, nuclear, accelerator, and space physics.

\section{BACKGROUND}

\section{A. Reflectance measurements}

We have previously designed and built an instrument to accurately measure the angular reflection distribution inside of a crystal [12]. The principle of our instrument is illustrated in Fig. 1. An incident beam is produced by a $440-\mathrm{nm}$ laser, which can be positioned at any theta $(\theta)$ angle. The angle phi $(\phi)$ is defined to be perpendicular to the plane the laser moves in (the $\theta$-plane), along the detector arch. The reflected light is detected by an array of 36 PIN photodiodes, mounted in two rows on an arch. The detector arch moves from $\theta$ equal to $-90^{\circ}$ to $+90^{\circ}$, covering the full $2 \pi$ of solid angle. All light entering and exiting the crystal is perpendicular to the hemispherical surface, enabling all reflected light to be measured, as there is no total-internal-reflection.

The instrument has been used to measure the reflectance of BGO crystals with polished, etched, and rough-cut surfaces, and various reflectors $\left(\right.$ Teflon $^{\circledR}$ tape, ESR film, Lumirror ${ }^{\circledR}$, $\mathrm{TiO}_{2}$ paint, and Tyvek ${ }^{\circledR}$ paper) attached, both air-coupled and 
glued [11]. Each of these surface-reflector measurements consist of a set of angular reflection distributions produced by a set of laser incidence angles. Each of these angular distributions has a resolution of $4^{\circ} \times 5^{\circ}$ in theta and phi respectively. One such angular distribution measurement (for one incidence angle) is illustrated in Fig. 2. This set of measurements is the basis for our simulations.

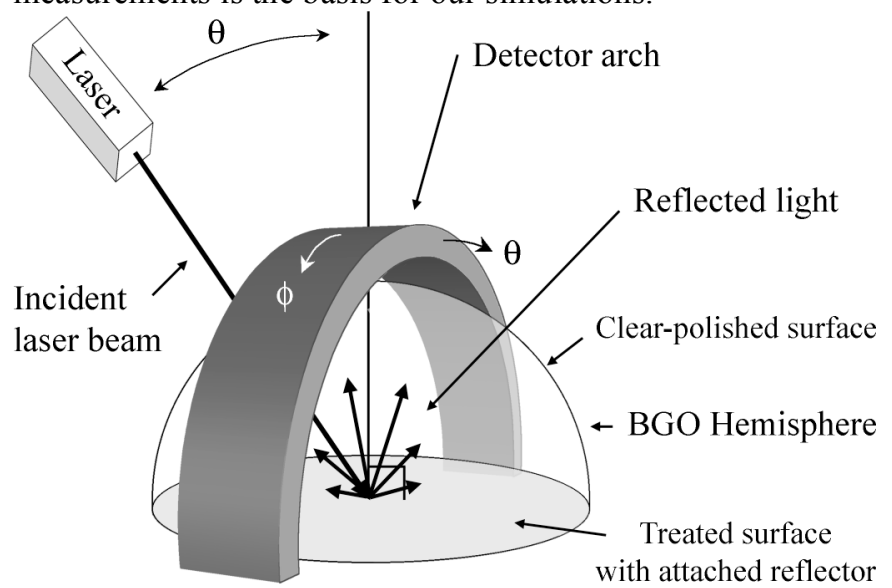

Fig. 1. Sketch to illustrate the principle used for measuring reflectance distributions. An incident laser beam is reflected off the bottom (flat) surface of a BGO hemisphere, and the reflected light is measured by an array of PIN photodetectors, which are mounted on an arch. The detector arch moves from theta $-90^{\circ}$ to $+90^{\circ}$, thus enabling measurements of the full $2 \pi$ of solid angle. All light entering and exiting the hemisphere is perpendicular to the surface, thus enabling all reflection angles to be measured. The laser is mounted on the outside of the detector arch and is movable from theta $-90^{\circ}$ to $+90^{\circ}$, with $\mathrm{phi}=$ $0^{\circ}$.

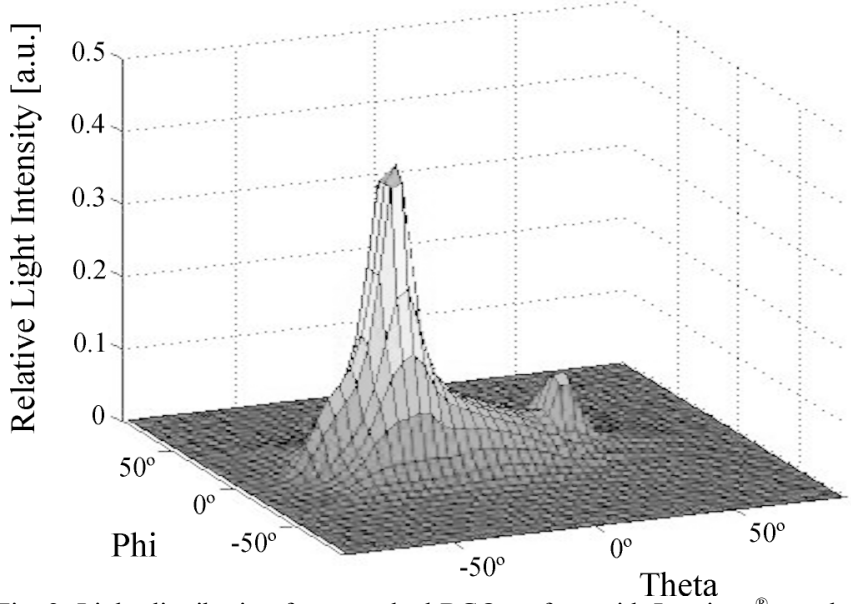

Fig. 2. Light distribution for an etched BGO surface with Lumirror ${ }^{\circledR}$ attached at laser incidence angle of $26^{\circ}$. A reflection peak is seen at $\theta=-26^{\circ}$, and a secondary reflection peak is seen at $\theta=26^{\circ}$.

\section{B. Current Monte Carlo codes}

The current Geant 4 code (version 9.2) allows the user to select between two optical reflection models - the glisur model and the unified model. The glisur model assumes that the surface is made up of micro-facets, where a micro-facet is selected from a distribution each time a reflection occurs. The micro-facet normal is calculated as the sum of two vectors; the average surface nominal normal, and a second vector, which is defined by a random point on a sphere of radius $(1-$ polish $)$, with polish $<1$, and added to the tip of the first vector. A specular reflection is thereafter calculated based on the micro- facet orientation. The unified model is also based on microfacets, and since the current GATE code - which calls the Geant 4 code - is hardwired to the unified model, we will concentrate in this section on describing the main features of the unified model.

In the unified model, four kinds of surface reflections are possible: specular spike, specular lobe, backscatter, and Lambertian. For the specular spike reflection, the reflected photon is reflected about the average surface normal, in the same way a photon would be reflected in a perfect mirror. For backscatter reflection, the photon is reflected back into the direction the photon came from. In Lambertian reflection, the photon will be reflected with a Lambertian distribution probability, i.e., into a cosine distribution around the average surface normal.

In the unified model and for a specular lobe reflection, the surface is assumed to consist of microscopic small surfaces, or micro-facets, which are oriented around the average surface (i.e., the macro-surface normal) with a Gaussian distribution, see Fig. 3. A sigmaalpha parameter defines the standard deviation of the distribution of the micro-facets orientations. Each time a specular lobe interaction occurs, a micro-facet is randomly selected from the distribution that is defined by sigmaalpha, and a specular reflection is thereafter calculated based on this micro-facet orientation.

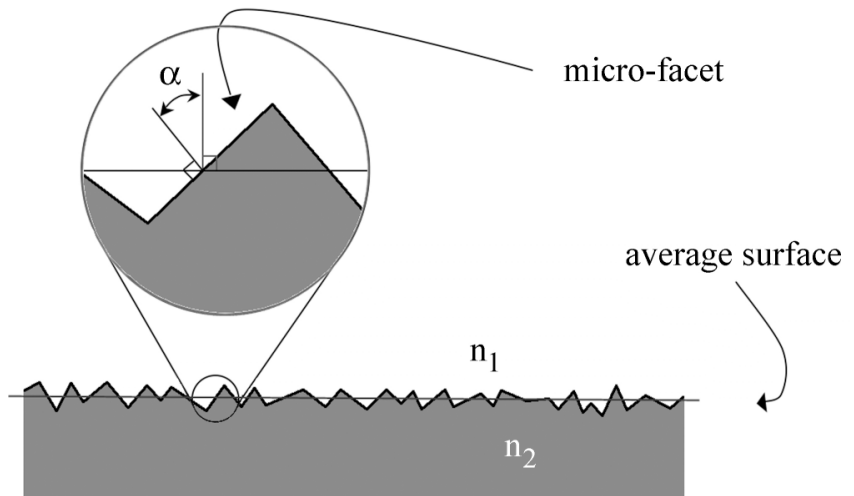

Fig. 3. For a ground surface in the unified model, the parameter sigmaalpha defines the standard deviation of the Gaussian distribution of micro-facets around the average surface normal.

For an optical simulation to be performed in GATE, the user has to define the surface by selecting the surface type (i.e., dielectric-to-dielectric or dielectric-to-metal), the surface finish (i.e., polished or ground), the refractive indices for the two materials defining the surface, the reflectivity of the reflector attached to the surface (which can either be set as a Lambertian or a specular reflector), and the probabilities for each of the four surface reflection types. The probabilities for these four reflection types must add up to $100 \%$ of the reflected light, and the relative probabilities can be very hard for the casual user to estimate. In addition, if the user opts to use the specular lobe reflection distribution, the user also has to set a value to the surface distribution parameter sigmaalpha, something that is very difficult for most users to estimate (even if they have a way of measuring it - more on this in the discussion section).

One important observation is that the unified model in Geant 4 assumes that the four reflection type probabilities are constants, and not functions of incidence angle. This does not 
fully agree with our measured data, as for instance a Lambertian reflector (e.g., Teflon ${ }^{\circledR}$ tape) attached to a BGO surface exhibits Lambertian and specular lobe reflection distributions for low incidence angles and a specular lobe (turning into a specular spike) reflection distribution for high (very high) incidence angles [11]. The Lambertian portion of the reflection distribution at low incidence angles is most likely due to the Lambertian reflector underneath the surface, and the surface produces the specular lobe reflection distribution. At very high angles however, the specular lobe reflection distribution becomes narrower, asymptotically approaching a specular spike reflection distribution. If the specular lobe portion of the reflected light truly was independent of incidence angle as the unified code assumes, the specular lobe would maintain its shape for these very high angles, at least for lower values of sigmaalpha, and not narrow into a specular spike. This discrepancy is also observed for reflectors assumed to be Lambertian, where the fraction of Lambertian reflection distribution decreases with increasing incidence angle in our measured angular distributions [10].

\section{METHODS}

\section{A. Mathematical corrections to the measured data}

The measured data was acquired from laser incidence angles in $4^{\circ}$ steps, from $2^{\circ}$ to $82^{\circ}$ [11]. This data needed to be resampled to every whole angle, including the endpoints of perpendicular incidence $\left(\theta=0^{\circ}\right)$ and parallel incidence $\left(\theta=90^{\circ}\right)$, i.e., $\theta=\left[0^{\circ}: 1^{\circ}: 90^{\circ}\right]$, for accurate simulations. As previously described [11], one of the limitations with our instrument lies in that it cannot measure specular-like reflectance for $<10^{\circ}$ incidence angles, as the photodiode detectors at these low $\theta$-angles block the incident laser light. Therefore, we used the angular distribution for the lowest incidence angle we were able to measure to create a negative incidence angle distribution, and then used linear interpolation to estimate the distribution for perpendicular incidence. The parallel incidence endpoint was estimated to be purely specular (i.e., specular spike) from the high incidence angle distributions. With the endpoints in place, we re-sampled the angular distributions to every whole incidence angle with linear interpolation. The re-sampled angular distributions were thereafter corrected by subtracting secondary and higher order reflections from the data. The angular distributions were then corrected for solid angle uniformity, that is, the LUT values were multiplied by $\cos (\phi)$ to create a uniform solid angle distribution for our algorithm. The distributions were finally normalized and all values below $0.1 \%$ of the maximum value were set to zero to minimize the noise in the data. The final data was written to look-up-tables (LUTs) which the Monte Carlo code can access.

\section{B. Monte Carlo code changes}

We modified the Geant4 code to allow our LUTs to be used along with the existing surface reflection models. (No modifications were made to any transmission codes.) We had to introduce a new model, a new surface type, and new surface finishes. The user still has to set many of the parameters that are set in the unified model; however, the surface distribution

parameter sigmaalpha is no longer needed and neither are the four reflection type probabilities. GATE, which is an acronym for Geant4 Application for Emission Tomography, calls the Geant 4 code for the physics modeling, and therefore needs its modifications to be made in the Geant 4 code, and only minor modification need to be made to the GATE code. The unified model is automatically selected as the optical simulation model for GATE, and we therefore had to modify the GATE code to allow our new model with its parameters to be set for Geant4.

Our model works in principle as follows; when a photon encounters a surface, a random number (between 0 and 1) is generated. If this number is lower than the reflection coefficient, the photon is reflected; otherwise the photon is absorbed. (Up to this point, our model is identical to the glisur and unified models.) If the photon is reflected, the incidence angle is calculated, rounded to closest integer, and a random piece of solid angle (denoted by $\theta$ and $\varphi$ ) is chosen. The corresponding surface-type/reflector-LUT with the corresponding integer incidence angle is accessed (e.g., etched-Lumirror $^{\circledR}$-air-coupled at $17^{\circ}$ incidence), and the normalized probability for the $\theta$ and $\varphi$ angles is looked up in the LUT. A random number (between 0 and 1) is generated, and if this number is smaller than the LUT probability, the $\theta$ and $\varphi$ angles are accepted; otherwise new random $\theta$ and $\varphi$ angles are generated. This will repeat until a valid pair of $\theta$ and $\varphi$ angles is selected. With the new $\theta$ and $\varphi$ angles, a new photon direction is calculated. A new photon polarization is calculated by constructing a micro-facet normal from the old and new photon directions and, in the same way as the unified code, reflecting the photon's polarization in this micro-facet to get the new polarization vector.

a)

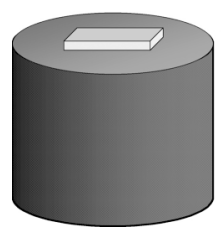

Fig. 4. The same crystal is positioned in three different orientations onto a PMT to validate the results of the simulations. By using the same crystal to measure ratios of the light output in three different orientations - through the large surface (a), the medium surface (b), and the small surface (c) - we avoid crystal-to-crystal variations in light output and get self-calibrated measurements.

\section{Choosing a validation setup}

To be able to compare the unified Monte Carlo code simulations with our LUT-simulations, we needed a validation method. We chose to validate the codes against light output measurements from a BGO crystal coupled in three different orientations to a photomultiplier tube (PMT), see Fig. 4. The BGO crystal has different lengths on each side, creating three different surface sizes that the scintillation light can be collected from. By measuring the same crystal in three different orientations, and normalizing the light output measurements to the large surface light output, we get lightratio measurements that are self-calibrating as crystal-tocrystal variations in the absolute light output are avoided. One 
crystal surface needs be grease-coupled to the PMT, while the remaining five surfaces will have reflector materials attached (Lumirror $^{\circledR}$, Teflon ${ }^{\circledR}$ tape, $\mathrm{TiO}_{2}$ paint, Tyvek ${ }^{\circledR}$ paper, or ESR film), either air-coupled or glued (using MeltMount ${ }^{\mathrm{TM}}$ ).

Preliminary simulations with our code implemented within the GATE and Geant4 codes were performed with specular surface settings and with diffuse surface settings to determine what crystal sizes to use. The criteria for size selection were based on: 1) achieving the largest possible light ratios out of the different sides of the crystal, and 2) having the smallest and largest crystal dimensions reasonable in regard to the experimental setup onto a PMT. That is, the largest surface length of the crystal must be smaller than $50 \mathrm{~mm}$ to fit onto a 2-inch diameter PMT window, and the smallest dimension of one crystal side cannot be smaller than a few millimeters to be able to securely couple the crystal to the PMT.

The light output from the BGO crystals were measured with a 51-mm diameter Hamamatsu R6231 PMT. The PMT surface was horizontal, and the uniformity of the PMT across the entire photosensitive area was measured in the $\mathrm{x}$ and $\mathrm{y}$ directions in $5 \times 5-\mathrm{mm}$ steps to make sure that the light output variations of the different crystal surface sizes are not caused by the PMT non-uniformity.

To reduce statistical error, we used five BGO crystals of each surface type (ground, chemically etched, and mechanically polished) from Hilger Crystals [Margate, Kent, UK] and Proteus, Inc. [Chagrin Falls, OH]. The crystals were irradiated with $511 \mathrm{keV}$ gammas from a Ge-68 source. The light output peak position was averaged for the five samples, and errors were estimated by computing the standard deviation for each surface type and attached reflector.

\section{Setting the parameters for standard Geant 4}

The reflectivity for the various reflectors was set according to Table I. We used literature values for the reflection coefficient for available referenced materials, and used our measured values for the remaining reflectors [10]. The reflection coefficients could theoretically change with incidence angle for the modified code since we are treating the surface and reflector as a single entity and there could be a higher degree of total-internal-reflection above the critical angle and a higher degree of transmission below the critical angle. However, measurements show that the total reflection coefficient varies very little with incidence angle when attaching a $95-99 \%$ reflector underneath the scintillator surface. We measured a total reflectance dependence on the incidence angle within a few percent in our angular distribution measurements (see Table III in [11]), validating the use of constant reflection coefficients to be used for the simulations. For the PMT we assumed a reflectivity of 0.20 and quantum efficiency of 0.25 . The crystal was optically coupled to the PMT with silicone grease. Table II displays the refractive indexes that were used for the various optical materials.
As previously mentioned, GATE and Geant 4 require the micro-facet distribution parameter sigmaalpha to be specified. We used our measured light distributions [11] to estimate sigmaalpha by determining the FWHM of the reflection peak above the critical angle, by assuming the peak distribution is Gaussian (i.e., $\mathrm{FWHM}=2.35 \cdot \sigma$ ), and by assuming the light distribution is twice the value of the micro-facet distribution. The FWHM of the light distribution for the various surface treatments was estimated to be $6^{\circ}$ for polished surfaces, $18^{\circ}$ for etched surfaces, and $<58^{\circ}$ for ground surfaces. Hence, sigmaalpha was set to be $1.3^{\circ}$ for polished surfaces, $3.8^{\circ}$ for etched surfaces, and $12^{\circ}$ for ground surfaces, respectively, see Table III. We also used a Dektak 150 surface profilometer [Veeco Instruments Inc., Plainview, NY] to produce surface profiles of the ground, etched, and polished surfaces. The surfaces were sampled every $0.056 \mu \mathrm{m}$ over 5 - $\mathrm{mm}$ lengths, using a $12.5-\mu \mathrm{m}$ radium stylus. The profilometer measurements produced surface profiles, which were used to calculate slope distributions for the polished, etched, and ground surfaces, see Fig. 5. The slope distribution data was fitted with Gaussian curves to estimate the parameter sigmaalpha. The polished surface slope distribution is described with sigmaalpha equal to $0.31^{\circ}$, while the etched and ground surfaces are not well described by Gaussian distributions.

TABLE I

REFLECTION COEFFICIENTS USED

\begin{tabular}{lcc}
\hline Reflector & Reflection coeff. & Source \\
\hline \hline Teflon $^{\circledR}$ & 0.99 & {$[13-15]$} \\
\hline ESR film & 0.985 & $\begin{array}{c}\text { 3M } \\
\text { St. Paul, MN }],[16]\end{array}$ \\
\hline Titanium dioxide paint & 0.955 & $\begin{array}{c}\text { [aint-Gobain Crystals } \\
\text { Newbury, OH }\end{array}$ \\
\hline Lumirror $^{\circledR}$ & 0.98 & {$[10]$} \\
\hline Tyvek $^{\circledR}{ }^{\circledR}$ paper & 0.97 & {$[10]$} \\
\hline \hline
\end{tabular}

TABLE II

REFRACTIVE INDEXES USED

\begin{tabular}{lcc}
\hline \hline Material & Refractive index & Source \\
\hline \hline BGO & 2.1 & [Preset in Geant4] \\
\hline Titanium dioxide paint & 1.61 & {$[11]$} \\
\hline MeltMount $^{\mathrm{TM}}$ & 1.582 & $\begin{array}{c}\text { [argille Laboratories } \\
\text { Ceder Grove, NJ }\end{array}$ \\
\hline Optical grease & 1.4042 & $\begin{array}{c}\text { GE Bayer Silicones } \\
\text { Albany, NY }\end{array}$ \\
\hline \hline
\end{tabular}

TABLE III

SIGMA-ALPHA

\begin{tabular}{ccc}
\hline \hline Polished & Etched & Ground \\
\hline \hline $1.3^{\circ}$ & $3.8^{\circ}$ & $12^{\circ}$ \\
\hline \hline
\end{tabular}

The unified model was used with the reflection type set to $100 \%$ specular lobe distribution. The surface type was set to dielectric_dielectric for all reflectors except for the ESR film and the PMT, where it was set to dielectric_metal. The surface 

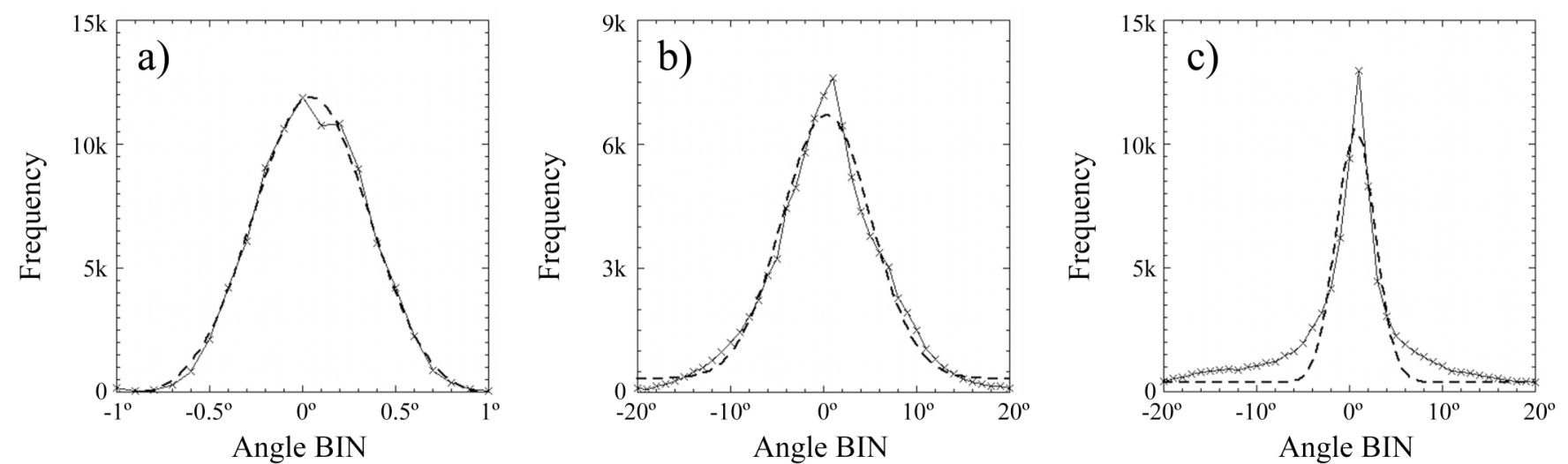

Fig. 5. Slope distributions for mechanically polished (a), chemically etched (b), and ground (c) surfaces. Gaussian fits (dashed curves) have been applied to the measured data (solid curves with hash marks). The standard deviation $(\sigma)$ for the Gaussian fits are $0.31^{\circ}$ for the polished surface (a), $4.76^{\circ}$ for the etched surface (b), and $2.03^{\circ}$ for the ground surface (c).

finish was set to groundbackpainted for all materials except for the ESR film and the PMT, where it was set to ground. (The reflection type defines the surface reflection, whereas the dielectric_dielectric with groundbackpainted defines a Lambertian reflector attached to the surface, and dielectric_metal with ground defines a specular reflector attached to a transparent surface, respectively.) For glued and painted reflectors, as well as the optical grease coupled PMT, the refractive index (RINDEX) was set according to Table II, and for all air-coupled reflectors the RINDEX interface was set to 1.0 , respectively. The reflectivity was set according to Table I, and sigmaalpha was set according to Table III. The crystal was assumed to be grease-coupled to the PMT.

The BGO crystal was modeled as having an optical absorption length of $1 \mathrm{~m}$, a scintillation yield of 8,200 photons / MeV, a refractive index according to Table II, and an energy resolution of $10 \%$ full-width-at-half-maximum (FWHM). For each setup, the crystal was irradiated with a $511 \mathrm{keV}$ gamma beam, parallel to the PMT surface and illuminating the center of the crystal. All simulations were performed with roughly 10,000 gammas impinging onto the crystal.

\section{E. Setting the parameters for our code}

Our model used the same values as the standard Geant4 code for the reflectivity and had the following choices for surface finish: polishedlumirrorair, polishedlumirrorglue, polishedteflonair, polishedtioair, polishedtyvekair, polishedvm2000air, polishedvm2000glue, etchedlumirrorair, etchedlumirrorglue, etchedteflonair, etchedtioair, etchedtyvekair, etchedvm2000air, etchedvm2000glue, groundlumirrorair, groundlumirrorglue, groundteflonair, groundtioair, groundtyvekair, groundvm2000air, or groundvm2000glue. The surface type was set to our newly defined surface type, as previously described in section III.B. The crystal was assumed to be grease-coupled to the PMT with the unified model as described above.

\section{RESULTS}

The crystal size for our validation measurements and for our simulations was set to $3 \times 10 \times 30 \mathrm{~mm}^{3}$. These dimensions were determined from our preliminary simulations, as shown in Fig. 6, along with the maximum and minimum size restriction criteria previously discussed in section III.C.

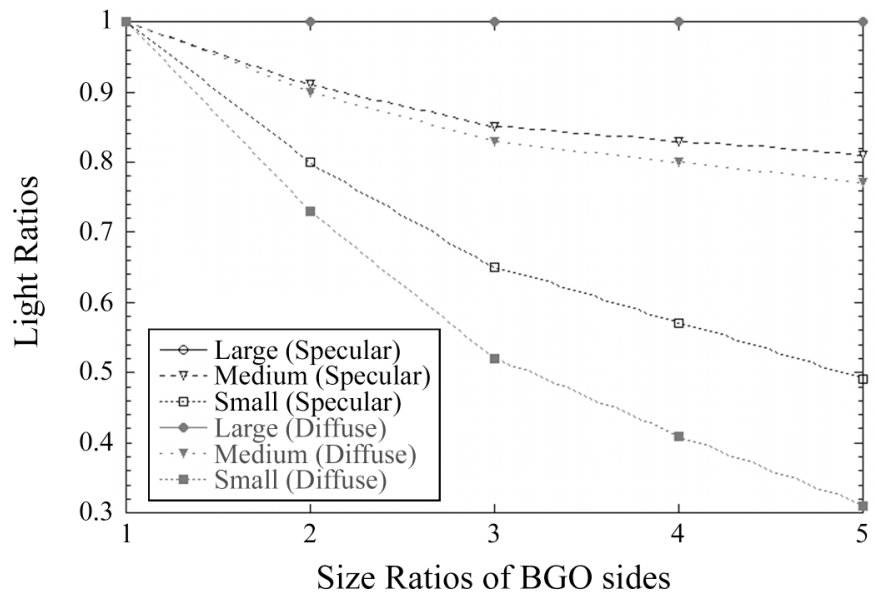

Fig. 6. Simulated light output from three surfaces of a BGO crystal. The specular simulations were performed with our code and air-coupled ESR film to a polished surface. The diffuse simulations were performed with our code and a $\mathrm{TiO}_{2}$-painted etched surface. The reflectivity was set to 0.98 in all simulations.

The PMT sensitivity was determined to be most uniform if the $3 \times 10 \times 30 \mathrm{~mm}^{3}$ crystals were be placed with their longest axis along the PMT $y$-direction. For this setup, the photosensitive uniformity of the PMT across the three surface dimensions $\left(3 \times 10 \mathrm{~mm}^{2}, 3 \times 30 \mathrm{~mm}^{2}\right.$, and $\left.10 \times 30 \mathrm{~mm}^{2}\right)$ was determined to be $\leq \pm 0.6 \%$.

The measured and simulated light ratios are displayed for polished, etched, and ground surfaces in Figs. 7-9. Fig. 7 displays the light ratios for polished surfaces, Fig. 8 displays the light ratios for etched surfaces, and Fig. 9 displays the light ratios for ground surfaces, respectively. Each figure consists of seven sets of columns, which correspond to the seven reflector material attachment combinations (which are labeled underneath each subset). Each of these sets consists of three columns, where the leftmost represent the measured data (with the error bars representing the standard deviation), the middle represent the results from our code simulations, and the rightmost represent the results from the original code simulations. Each of these columns in turn consists of two parts, where the striped part represents the light collected from the medium surface normalized to the light collected from the large surface, and the solid color part represents the light collected from the small surface normalized to the light collected from the large surface. 


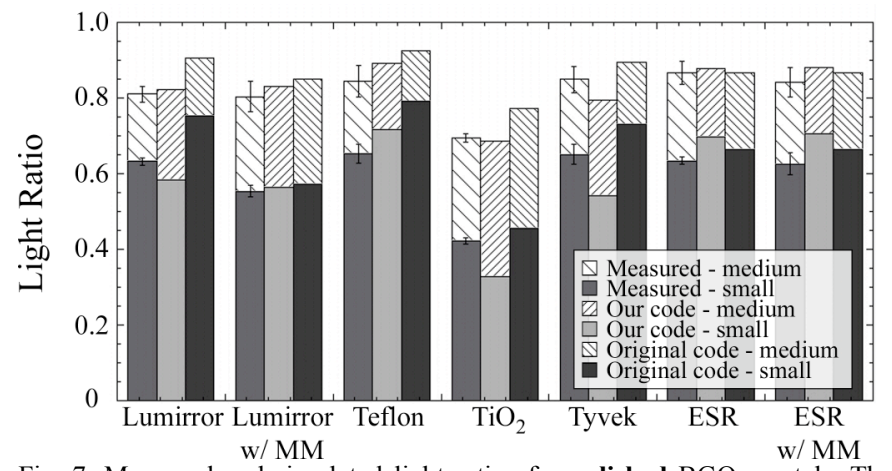

Fig. 7. Measured and simulated light ratios for polished BGO crystals. The measured data (with standard deviation error bars) is the leftmost columns, our code simulations are the middle columns, and the original code simulations are the rightmost columns for each attached reflector material. The striped columns show the light collected from a medium surface normalized to the light collected from a large surface, while the solid color columns show the light collected from a small surface normalized to the light collected from a large surface. The abbreviation "w/ MM" stands for "with MeltMount ${ }^{\mathrm{TM}}$,

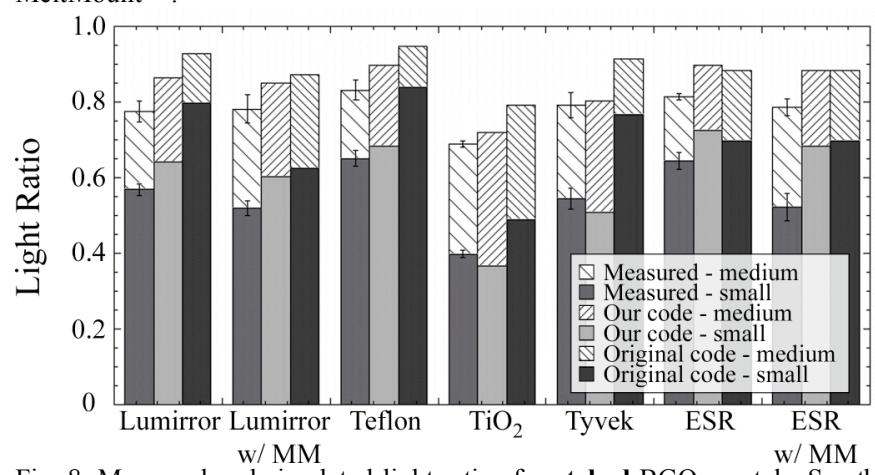

Fig. 8. Measured and simulated light ratios for etched BGO crystals. See the text for Fig. 7 for detailed description of the figure.

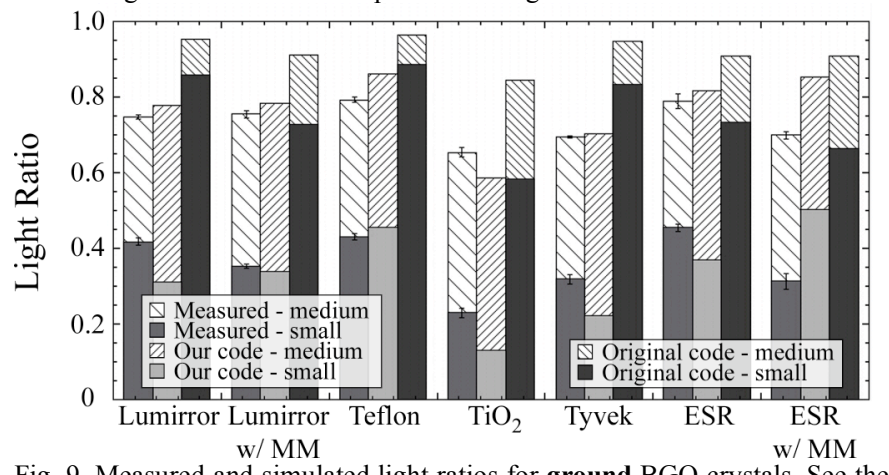

Fig. 9. Measured and simulated light ratios for ground BGO crystals. See the text for Fig. 7 for detailed description of the figure.

\section{Discussion}

The surface slope distribution measured with the profilometer in Fig. 5a estimates the parameter sigmaalpha for a polished surface to be $0.31^{\circ}$, which is slightly lower than the $1.3^{\circ}$ result from our measured optical angular distribution estimate (Table III). The difference between these two results (i.e., $1^{\circ}$ ) is within our error estimate for our setup [12]. The profilometer estimate is most likely the more accurate value as the profilometer has a higher degree of resolution compared to our $4^{\circ}$ by $5^{\circ}$ angular distribution measurements. However, we chose to use the value obtained by the optical method in our simulations, since the etched and rough surface estimates could (with less imagination compared to the profilometer results) be interpreted as Gaussian-distributed for the angular measurements, and for consistency. We ran simulations for several cases with sigmaalpha set to $0.31^{\circ}$ (versus $1.3^{\circ}$ ) and found the difference to be small (between $<1 \%$ and $5 \%$ ), and therefore include only simulation values for sigmaalpha equal to the $1.3^{\circ}$ in this paper. The fact that these two methods for obtaining sigmaalpha give different results highlights the difficulty for users to accurately simulate their surface / reflector properties in the current Geant4 code.

Lumirror $^{(B)}$ and Tyvek $\left.{ }^{(}\right)$are two reflector materials that are even more difficult to accurately simulate with the existing Geant 4 code, as these reflectors cannot be expressed as purely Lambertian or specular reflectors [10] as is required by the unified model in Geant4. For the unified code simulations we therefore had to approximate these two reflectors as purely Lambertian reflectors. For polished surfaces, where the choice of attached reflector has the largest impact [11], the Lambertian approximations seem to have been fairly accurate for the Tyvek ${ }^{\circledR}$ paper simulations, but not very good for the Lumirror $^{\mathbb{B}}$ simulations, as can be seen in Fig. 7. With our new simulation code, these approximations are no longer necessary, as we have introduced these reflector material's angular distributions into the code.

Both our model and the unified model are fairly accurate in predicting the light collected from polished BGO crystal surfaces, as can be seen in Fig. 7. Our model outperforms the unified model for Lumirror ${ }^{\circledR}$ (both air-coupled and glued) and Teflon ${ }^{\circledR}$ tape. On the other hand, the unified model has a slight edge over our model for ESR reflector covered surfaces. This is an expected result, as polished surfaces with ESR film are the easiest to model since the optical reflection model is specular for all incidence angles, and our code uses LUTs that have a lower angular resolution compared to the unified code. We expected the unified model to also be very accurate for Lambertian reflectors attached to polished surfaces, as this is the other "easy" simulation setup (i.e., a Lambertian reflector attached to specular surface). Although the light collection for titanium dioxide painted surfaces was fairly well predicted, it was not very accurate for the Teflon ${ }^{\circledR}$-wrapped surfaces. One possible explanation for this is that the optical reflectance model does not take into account that the four reflection type probabilities are functions of incidence angle, as previously mentioned in section II.B. In reality, reflection distributions $d o$ change with reflection angle as we have previously demonstrated [10], [11], in this particular case for the reflector (i.e., the Lambertian portion of the angular distribution deceases with increasing incidence angle).

For etched surfaces, see Fig. 8, the differences between our model and the unified model are more pronounced, especially for air (as opposed to MeltMount ${ }^{\mathrm{TM}}$ ) coupling. Our model outperforms the unified model for all attached reflectors but one - air-coupled ESR film - where the difference is minimal. For ground surfaces, see Fig. 9, the differences between our code and the unified code are substantial. For example, the unified code typically predicts a light ratio between the small and large surfaces that is between 0.7 and 0.9 , while our code predicts ratios that are much closer to the measured values, 
which are typically between 0.3 and 0.5 . Our code outperforms the unified code for all attached reflectors and agrees with the measured light collection very well, except for glued ESR film.

These two surfaces, etched and ground, are the hardest to simulate with the unified code for several reasons; 1) the casual user has difficulties estimating the parameter values for the simulation, but more importantly, 2) even if the user has the required knowledge about micro-facet orientation and reflection type probabilities (as we had, by measuring the parameters), the optical models are simply not very accurate. The parameter sigmaalpha assumes the macro-surface can be modeled as Gaussian distributed micro-facets, as was previously described in section II.B. In Fig. 5, we see that this might be a good model for the mechanically polished surface, but for the etched and ground surfaces the slope distributions do not follow Gaussian distributions. In addition, the actual reflection distributions depend on the angle of incidence, which is not included in the unified model. Thus, the surface reflection model used by the unified code has known inaccuracies. A scintillating photon will undergo many reflections before escaping the crystal and being detected, and even small errors in the angular distribution will therefore be amplified, ultimately leading to a large error. The optical models currently used by the Geant 4 code are mathematical models of the real world, but unfortunately not very accurate for more complicated surfaces as they do contain approximations and non-trivial errors. Using measured angular reflection distributions for surfaces that are not polished proved to be a much more accurate way of predicting the light collection of the PMT.

For polished surfaces, the execution time for the modified code was, depending on what reflector was attached, $150 \%$ to $290 \%$ of the original Geant4 code's execution time. This increase in execution time can easily be understood by realizing that a polished surface will produce mostly specular reflections, which are sparse LUT matrices, and our code will need more time to find a valid solid angle to reflect into. For etched surfaces, we measured an execution time for our code between $82 \%$ and $130 \%$ (depending on attached reflector) compared to the execution time for the original Geant4 code. For ground surfaces, the execution time for our code was between $83 \%$ and $120 \%$ (depending on attached reflector) compared to the original Geant 4 code. The simulation times for the modified code are thus comparable to the original Geant4 simulation times for the cases where our code outperforms the original code - for non-polished surfaces.

Although ground surfaces can be prepared many different ways (e.g., saw-cut, 40-grit sandpaper, sand blasted, aluminum oxide slurry polished), the reflected light distribution for them seems to be fairly similar. In [11], we measured the angular reflection distribution for a ground surface from a saw-cut surface. The $3 \times 10 \times 30 \mathrm{~mm}^{3}$ BGO crystals that were delivered to us had much finer saw-cut surfaces (i.e., more uniform) than the hemispheres', and although the hemisphere and the $3 \times 10 \times 30 \mathrm{~mm}^{3}$ crystal surfaces were not identical, the LUTs acquired from the hemisphere produced accurate estimates of the light collection, as can be seen in Fig. 9, validating this assumption. The assumption was also verified by the profilometer measurements, as the two ground surfaces, as well as a 60-grit sandpapered BGO surface, all produced very similar slope distributions.

As a bonus feature to making the optical simulation codes GATE and Geant4 more accurate, our new code is also more user friendly; the only required knowledge from the user for our code is the surface roughness (i.e., mechanically polished, chemically etched, or ground), the reflector attached (i.e., Teflon $^{\circledR}$ tape, ESR film, Lumirror ${ }^{\circledR}, \mathrm{TiO}_{2}$ paint, and Tyvek ${ }^{\circledR}$ paper), and how the reflector is attached (i.e., air-coupled or glued).

\section{CONCLUSIONS}

We have made modifications to the standard GATE and Geant4 simulation toolkits to be able to simulate light reflectance with measured data. Our new model is more accurate as well as more user friendly. Our code shows better agreement with the measured data than the standard Geant4 code, and our code also allows more reflector materials to be simulated. No detailed knowledge about the surfaces or light distributions is required from the user for our code; the user is only required to know the surface type and what reflector is attached. We are planning to make these modifications part of the standard GATE and Geant4 Monte Carlo packages in the very near future.

\section{ACKNOWLEDGMENT}

This work was supported by the National Nuclear Security Administration, Office of Defense Nuclear Nonproliferation, Office of Nuclear Nonproliferation Research and Engineering (NA-22) of the U.S. Department of Energy under Contract No. DE-AC02-05CH11231, grant number NNSA LB06-316-PD05 / NN2001000.

\section{REFERENCES}

[1] G.F. Knoll, T.F. Knoll, and T.M. Henderson, "Light collection in scintillating detector composites for neutron detection," IEEE Trans. Nucl. Sci., vol. 35, pt. 1, pp. 872-875, Feb. 1988.

[2] F. Cayouette, D. Laurendeau, and C. Moisan, "DETECT2000: An Improved Monte-Carlo Simulator for the Computer Aided Design of Photon Sensing Devices", Proc. SPIE, 2003, vol. 4833, pp. 69-76, doi: $10.1117 / 12.474315 .1117 / 12.474315$.

[3] F.-X. Gentit, "Litrani: a general purpose Monte-Carlo program simulating light propagation in isotropic or anisotropic media", Nucl. Instr. Meth. A, vol. 486, no. 1-2, pp., p. 35, Jun. 2002

[4] J. Allison et al., "Geant4 Developments and Applications", IEEE Trans. Nucl. Sci., vol. 53, no. 1, pp. 270-278, Feb. 2006

[5] S. Agostinelli et al., "Geant4 - A Simulation Toolkit", Nucl. Instr. Meth. $A$, vol. 506, pp. 250-303, 2003

[6] G. Santin, D. Strul, and D. Lazaro et al., "GATE: A Geant4-Based Simulation Platform for PET and SPECT Integrating Movement and Time Management", IEEE Trans. Nucl. Sci., vol. 50, no. 5, pp. 15161521, Oct. 2003

[7] D. Strul, G. Santin, and D. Lazaro et al., "GATE (Geant4 Application for Tomographic Emission): a PET/SPECT general-purpose simulation platform", Nucl. Phys. B (Proc. Suppl.), vol. 125, pp. 75-79, 2003

[8] K. Assie, V. Breton, and I. Buvat et al., "Monte Carlo simulation in PET and SPECT instrumentation using GATE”, Nucl. Instr. Meth. Phys. Res. $A$, vol. 527, pp. 180-189, 2004 
[9] S. Jan, G. Santin, and D. Strul et al., "GATE: a simulation toolkit for PET and SPECT”, Phys. Med. Biol., vol. 49, pp. 4543-4561, 2004

[10] M. Janecek and W.W. Moses, "Optical Reflectance Measurements for Commonly Used Reflectors", IEEE Trans. Nucl. Science, vol. 55, no. 4, pt. 2, pp. 2432-2437, Aug. 2008

[11] M. Janecek and W.W. Moses, "Measuring Light Reflectance of BGO Crystal Surfaces", IEEE Trans. Nucl. Science, vol. 55, no. 5, pt. 1, pp. 2443-2449, Oct. 2008

[12] M. Janecek and W.W. Moses, "Design of an Instrument to Measure Optical Reflectance of Scintillating Crystal Surfaces", IEEE Trans. Nucl. Sci., vol. 55, no. 3, pt. 2, pp. 1381-1386, Jun. 2008

[13] , "A Guide to Reflectance Coatings and Materials," Labsphere, Reflections Newsletter, pp. 8-11, Sep. 1998

[14] V.R. Weidner and J.J. Hsia, "Reflection Properties of Pressed Polytetrafluoroethylene Powder", J Opt. Soc. Amer., vol. 71, no. 7, pp. 856-861, 1981

[15] B. Waldwick, C. Chase, and B. Chang, "Increased efficiency and performance in laser pump chambers through use of diffuse highly reflective materials", in Proc. SPIE, 2007, vol. 6663, pp. 66630N.166630 N.7

[16] D. Motta, C. Buck, F.X. Hartmann, Th. Lasserre, S. Schönert, and U. Schwan, "Prototype scintillator cell for an In-based solar neutrino detector", Nucl. Instr. Meth. Phys. Res. A, vol. 547, pp. 368-388, 2005 